\title{
NOTES ON THE GENUS STICHORKIS (ORCHIDACEAE, MALAXIDINAE) IN MALESIA: NEW COMBINATIONS, SYNONYMS AND LECTOTYPIFICATIONS
}

\author{
Mark Arcebal K. Naive ${ }^{1,3}$ \& Paul Ormerod ${ }^{2}$ \\ ${ }^{1}$ Department of Biological Sciences, College of Science and Mathematics, \\ Mindanao State University-Iligan Institute of Technology, Andres Bonifacio Ave, Iligan City, \\ 9200 Lanao del Norte, Philippines \\ ${ }^{2}$ P.O. Box 8210, Cairns 4870, Queensland, Australia \\ ${ }^{3}$ Corresponding author email: arciinaive19@gmail.com
}

\begin{abstract}
Stichorkis are proposed. This work aimed to solve taxonomic ambiguity and nomenclatural crisis for Malesian Stichorkis. KEY wORDS: Malaxideae, new combinations, Orchidaceae, Philippines, plant Taxonomy, tropical botany
\end{abstract}

Introduction. The orchid genus Stichorkis Thouars (nom. \& typ. cons.) is represented by approximately 60 species distributed from the Comoro Islands, Mauritius, Reunion to Sri Lanka, India, through Malesia to Fiji with the centre of diversity in New Guinea where 23 species are recorded (Naive et al. 2019). In the process of examining all types of orchids in Malesia, in preparation for previous and ongoing revision, it became clear that more names than those published by Cootes (2011) need combination in Philippine Stichorkis. In this paper, we transfer five taxa from Cestichis Thouars ex Pfitzer and one from Liparis Rich., two of which had been overlooked. The other four had an incorrect basionym when previously transferred, therefore making the names either invalid or illegitimate (Article 41.6; Turland et al. 2018). Thus, the opportunity to emend the nomenclature is taken here. We also use the occasion to lectotypify these names, which is why type data are cited here. Furthermore, nine new combinations from West Malesia and 26 new combinations from East Malesia are proposed.

NeW COMBINATIONS AND A NEW SYNONYM FROM THE PHILIPPINES

Stichorkis elmeri (Ames) Naive \& Ormerod, comb. nov. Basionym: Cestichis elmeri Ames, Orchidaceae 1: 10. 1905.

Synonym: Liparis elmeri (Ames) Schltr., Repert.

Sp. Nov. Regni Veg., Beih. 1: 210. 1911.
Disticholiparis elmeri (Ames) Marg. \& Szlach., Orchidee (Hamburg) 55, 2: 178. 2004.

TYPE: PHILIPPINES. Luzon: Mt. Santo Tomas, Prov.

Benguet, "forming colonies on trees", 1 July 1904, A.D.E. Elmer 6553 (holotype: AMES00106597!; isotypes: K, US, NY). FIG. 1B.

Distribution: This Philippine endemic species is only recorded and observed in the provinces of Benguet and Quezon of Luzon island (Pelser et al. 2011).

Among the Malaxidinae herbarium specimens, especially at AMES, we found plants preliminarily called Cestichis elmeri. The plants are without any doubt is representative of the genus Stichorkis and is therefore recognized here as a new combination. According to Ames (1905), this species is closely similar to $S$. merrillii (Ames) Naive \& Ormerod by having an orbicular-apiculate labellum, non-emarginate anther, and laxer inflorescence. This taxon is not to be confused with Liparis elmeri Ames, described in 1912 and renamed $L$. dumaguetensis Ames. The latter is a terrestrial species with convolute leaves, unrelated to the genus Stichorkis.

Stichorkis gibbosa (Finet) J.J.Wood, Orchids Mount Kinabalu 2: 531. 2011.

Basionym: Liparis gibbosa Finet, Bull. Soc. Bot. France 342. 1908. Disticholiparis gibbosa (Finet) Marg. \& Szlach., Orchidee (Hamburg) 55: 178, 2004.

TYPE: INDONESIA. Java, without locality, Blume s.n. (holotype: P-image!) 
Liparis quadribullata Schltr., Repert. Spec. Nov. Regni Veg. 10: 29. 1911. TYPE: INDONESIA. Sulawesi, Toli-Toli District, upper Lampasioe River, 150 m, January 1910, Schlechter 20668 (holotype: B, destroyed; isotypes: AMES, K), syn. nov.

Distribution: Myanmar, Vietnam, Cambodia, Laos, Thailand, Peninsular Malaysia, Sumatra, Java, Borneo, Indonesia, New Guinea, Pacific Islands, Philippines.

Stichorkis gracilis (Ames) Naive \& Ormerod, comb. nov.

Basionym: Cestichis gracilis Ames, Orchidaceae 2: 136. 1908.

Synonyms: Liparis amesiana Schltr., Repert. Sp. Nov.

Regni Veg., Beih. 1: 210. 1911. Disticholiparis gracilis (Ames) Marg. \& Szlach., Orchidee (Hamburg) 55, 2: 178. 2004. Stichorkis amesiana (Schltr.) Cootes, Philipp. Nat. Orch. Sp.: 245. 2011, nom. illeg.

TYPE: PHILIPPINES. Luzon, Bataan Prov., Mt.

Mariveles, 31 January 1904, E.B. Copeland 274 (lectotype, here designated: AMES8217!; isolectotypes: K, US); Rizal Prov., Mt. San Isidro, 10 January 1907, M. Ramos 1782 (syntype: AMES-image!; isosyntype: K). FIG. 1C.

Distribution: Endemic to the Philippines. Recorded in the provinces of Bataan, Rizal, Mindoro, Bukidnon, and Davao.

Because of the earlier Liparis gracilis J.D. Hook. of 1890 (= L. elegans Lindl.) and L. gracilis Rolfe of 1891 (= L. gracilenta Dandy), Schlechter renamed Cestichis gracilis Ames as Liparis amesiana when he moved the plant to Liparis. Since the name Cestichis gracilis is the oldest and the epithet is still available, it is the one that must be used when treating the plant in Stichorkis. We have chosen Copeland 274 as lectotype since this has a copy of the original illustration appended to it by Ames.

Stichorkis merrillii (Ames) Naive \& Ormerod, comb. nov.

Basionym: Cestichis merrillii Ames, Orchidaceae 1: 11. 1905.

Synonyms: Liparis merrillii (Ames) Schltr., Repert.

Sp. Nov. Regni Veg., Beih. 1: 210. 1911.

Disticholiparis merrillii (Ames) Marg. \& Szlach.,

Orchidee (Hamburg) 55, 2: 178. 2004.
TYPE: PHILIPPINES, Luzon, Bataan Prov., Mt. Mariveles, 1200 m, 1 January 1905, E.D. Merrill 3736 (holotype: AMES00106604!; isotype: US 00093468). FIG, $1 \mathrm{D}$.

Distribution: Endemic to the Philippines. Recorded in the provinces of Bataan, Ifugao, Mountain Province, Nueva Ecija, Nueva Vizcaya, Quezon, Rizal, Mindoro, Leyte, Misamis, Zamboanga.

Though Ames (1905) listed two collections when he published this species, he implied in his notes that Merrill 3736 was the type, and that H.N. Whitford 317 (AMES) was a second collection, i.e. a paratype. Jim Cootes transferred this taxon to Stichorkis in 2011, but unfortunately cited the incorrect basionym (Liparis merrillii), thus making the proposal invalid.

Stichorkis nutans (Ames) Naive \& Ormerod, comb. nov.

Basionym: Cestichis nutans Ames, Philipp. J. Sci., C 4: 597. 1909.

Synonym: Liparis nutans (Ames) Ames, Orchidaceae 5: 81. 1915.

TYPES: PHILIPPINES. Mindanao, Lake Lanao, Camp Keithley, May 1907, M.S. Clemens s.n. (lectotype, here designated: AMES00106605!); Surigao Prov., 6 April 1906, P.H. Bolster 289 (syntype: AMES-image!); Palawan, Mt. Victoria, March 1906, F.W. Foxworthy s.n. (= BS 638) (syntype: AMES-image!). FIG. 1E.

Distribution: Endemic to the Philippines. Recorded in the provinces of Palawan, Agusan, Bukidnon, Lanao, Misamis Oriental, Surigao.

Like S. merrillii above this taxon had been transferred to Stichorkis but by incorrectly citing the later name in Liparis, making the proposal invalid. This species closely resembles $S$. davidlohmanii (Fig. 1A), however, it differs significantly in having a pyriform pseudobulb (vs. obovoid pseudobulb) and cuneate, non-canaliculate, conduplicate labellum (vs. obovate, canaliculate, non-conduplicate labellum).

Stichorkis philippinensis (Ames) Naive \& Ormerod, comb. nov.

Basionym: Cestichis philippinensis Ames, Orchidaceae 1: 7.1905. 
Synonym: Liparis philippinensis (Ames) Schltr., Repert. Sp. Nov. Regni Veg., Beih. 1: 210. Disticholiparis philippinensis (Ames) Marg. \& Szlach., Orchidee (Hamburg) 55, 2: 178. 2004.

TYPES: PHILIPPINES, Luzon, Bataan Prov., Mt. Mariveles, 25 May 1904, T.E. Borden 799 (lectotype, here designated: AMES5966!; isolectotype: US-image!); Mt. Mariveles, 8 August 1904, E.D. Merrill 3856 (syntype: AMES!; isosyntypes: K-image!, NY-image!); Mt. Mariveles, 9 August 1904, T.E. Borden 1597 (syntype: AMES-image!). FIG. 1F.

Distribution: Endemic to the Philippines. Recorded in the provinces of Abra, Bataan, Benguet, Cagayan, Laguna, Mountain Province, Nueva Vizcaya, Pampanga, Rizal, Mindoro, Panay, Leyte, Negros, Agusan, Bukidnon, Cotabato.

Again, as in some of the above names, the incorrect basionym was cited when transferring this taxon to Stichorkis, rendering the combination invalid.

Stichorkis propinqua (Ames) Naive \& Ormerod, comb. nov.

Basionym: Liparis propinqua Ames, Orchidaceae 7: 110. 1922

Synonym: Disticholiparis propinqua (Ames) Marg. \&

Szlach., Orchidee (Hamburg) 55, 2: 179. 2004.

TYPE: PHILIPPINES, Bancalan Island, sea level,

14 October 1916, C.M. Weber 011 (holotype:

AMES00100960!; isotypes: K, NY, S, SING!, US).

Distribution: Endemic to the Philippines. Recorded in the provinces of Bancalan Island, Davao, Laguna, Mindoro, Palawan, Quezon and Rizal.

NEW COMBINATIONS AND A NEW SYNONYM FROM West Malesian taxa

Stichorkis anopheles (J.J.Wood) Naive \& Ormerod, comb. nov.

Basionym: Liparis anopheles J.J.Wood, Nord. J. Bot. 11, 1: 85. 1991.

Synonym: Disticholiparis anopheles (J.J.Wood) Marg. \& Szlach., Orchidee (Hamburg) 55, 2: 176. 2004.

TYPE: MALAYSIA. Sabah, Tambunan District, Mt.

Trus Madi, above Kidukarok, 1560 m, 15 June 1988, Surat in J.J. wood 871 (holotype: K).

Distribution: Malaysia (Sabah).
Stichorkis araneola (Ridl.) Naive \& Ormerod, comb. nov.

Basionym: Liparis araneola Ridl., J. Linn. Soc., Bot. 31: 265. 1896.

Synonym: Disticholiparis araneola (Ridl.) Marg. \& Szlach., Orchidee (Hamburg) 55, 2: 176. 2004.

TYPE: INDONESIA. Kalimantan, Pontianak, cult.

Bot. Gard. Singapore s.n. (holotype: SING!).

Distribution: Indonesia (Kalimantan).

Stichorkis bibullata (J.J.Sm.) Naive \& Ormerod, comb. nov.

Basionym: Liparis bibullata J.J.Sm., Bull. Jard. Bot. Buitenzorg, sér. 3, 9: 143. 1927.

Synonym: Disticholiparis bibullata (J.J.Sm.) Marg. \&

Szlach., Orchidee (Hamburg) 55, 2: 176. 2004.

TYPE: SUMATRA. Aceh, Gajo Loees, Leg. Van

Daalen, cult. Hort. Bogor. 200 (holotype: BO).

DistRibution: Indonesia (Sumatra)

Stichorkis biglobulifera (J.J.Sm.) Naive \& Ormerod, comb. nov.

Basionym: Liparis biglobulifera J.J.Sm., Bull. Jard. Bot. Buitenzorg, sér. 3, 9: 143, 1927.

Synonym: Disticholiparis biglobulifera (J.J.Sm.) Marg. \& Szlach., Orchidee (Hamburg) 55, 2: 176. 2004.

TYPE: SUMATRA. Gunung Singgalang, $1900 \mathrm{~m}$, Leg. Groeneveldt, cult. Hort. Jacobson 1322 (holotype: $\mathrm{BO})$.

Distribution: Indonesia (Sumatra).

Stichorkis kemulensis (J.J.Sm.) Naive \& Ormerod, comb. nov.

Basionym: Liparis kemulensis J.J.Sm., Bull. Jard. Bot. Buitenzorg, sér. 3, 12: 149. 1932.

Synonym: Liparis amesiana J.J.Sm., Bull. Jard. Bot. Buitenzorg, sér. 3, 11: 124. 1931 nom. illeg. (non Schltr. 1911).

TYPE: INDONESIA. Kalimantan, West Koetai, Gunung Kemoel, 1500 m, 15 October 1925, Endert 4167 (holotype: L).

Distribution: Indonesia (Kalimantan).

Stichorkis kerintjiensis (J.J.Sm.) Naive \& Ormerod, comb. nov.

Basionym: Liparis kerintjiensis J.J.Sm., Bull. Jard. Bot. Buitenzorg, sér. 3, 10: 50. 1928. 


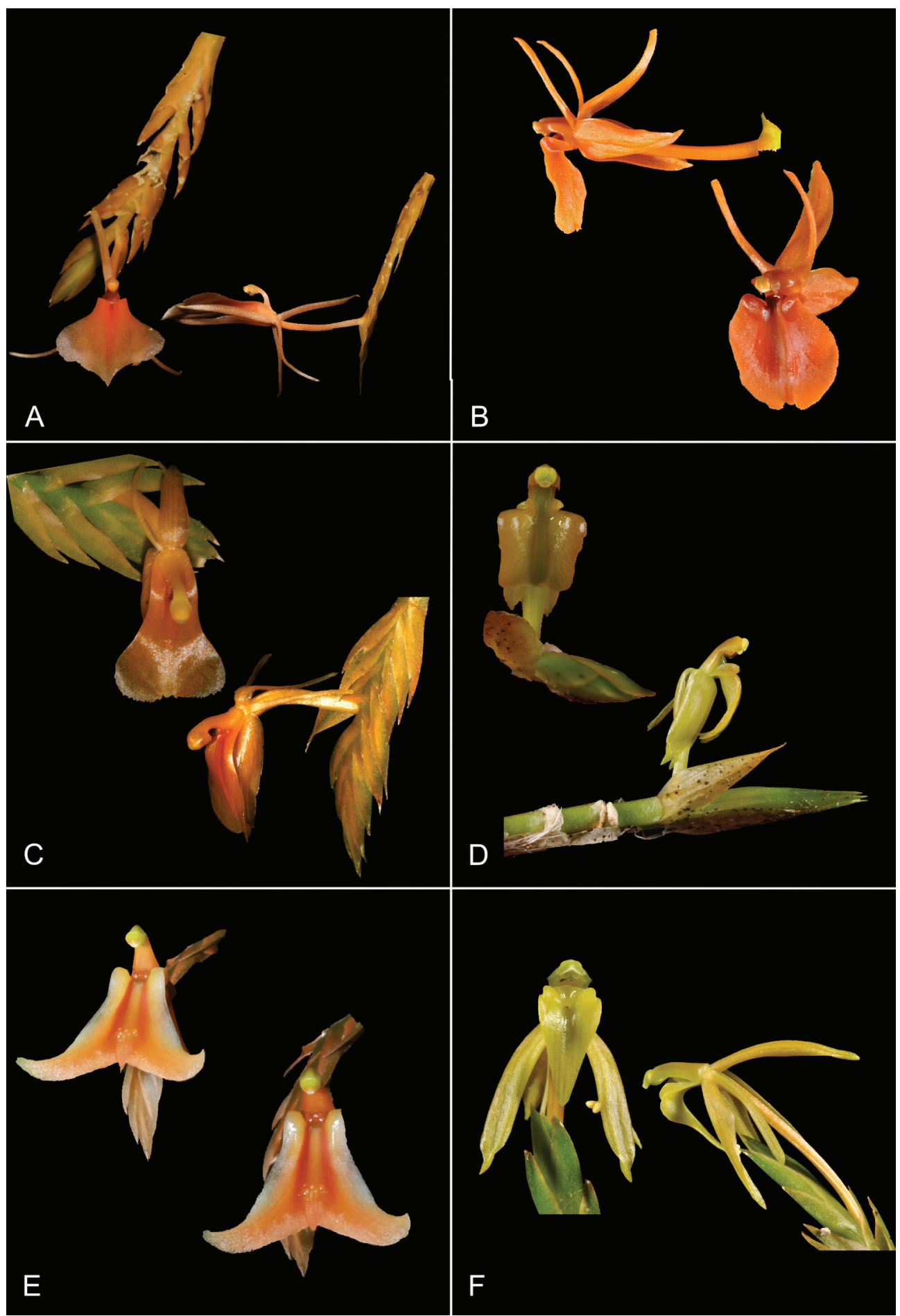

FIgURE 1. Selected Philippine Stichorkis species A. S. davidlohmanii B. S. elmeri C. S. gracilis D. S. merrillii E. S. nutans F. S. philippinensis. Photographs by: M. A. K. Naive (A, E), Ravan Schneider (C), Pieter Pelser \& Julie Barcelona (B, $\mathrm{D}, \mathrm{F})$. 
Synonym: Disticholiparis kerintjiensis (J.J.Sm.) Marg. \& Szlach., Orchidee (Hamburg) 55, 2: 178. 2004.

TYPES: INDONESIA. Sumatra, Gunung Kerintji, 1200 m, 8 March 1920, Bünnemeijer 8600 (syntype: BO); Gunung Kerintji, 1700 m, 16 March 1920, Bünnemeijer 8911 (syntype: BO; isosyntypes: AMES!, K, L); Gunung Kerintji, 1200 m, 17 March 1920, Bünnemeijer 8955 (syntype: $\mathrm{BO}$; isosyntype: AMES!).

Distribution: Indonesia (Sumatra).

Stichorkis lycopodioides (J.J.Sm.) Naive \& Ormerod, comb. nov.

Basionym: Liparis lycopodioides J.J.Sm., Bull. Jard.

Bot. Buitenzorg, sér. 3, 11: 121. 1931.

TYPE: INDONESIA. Kalimantan, West Koetai, Long

Petak, 450 m, 20 September 1925, Endert 3492 (holotype: L).

Distribution: Indonesia (Kalimantan).

Stichorkis mucronata (Blume) J.J.Wood, Orchids Mount Kinabalu 2: 532. 2011.

Basionym: Malaxis mucronata Blume, Bijdr. Fl. Ned. Ind.: 391. 1825. Liparis mucronata (Blume) Lindl., Gen. Sp. Orchid. P1.: 32. 1830. TYPE: INDONESIA. Java, mountain of Bantam Province and Buitenzorg Province, H. Kuhl \& J.C. van Hasselt s.n. (holotype: L-image!).

Liparis celebica Schltr., Repert. Sp. Nov. Regni Veg. 10:

28. 1911. TYPE: INDONESIA. Sulawesi, Minahassa Peninsula, Mt. Masarang, 1200 m, November 1909, Schlechter 20668 (holotype: B, destroyed; isotypes: AMES!, G, K, L, NSW!, S), syn. nov.

Distribution: Borneo, Jawa, Lesser Sunda Is., Sulawesi, and Sumatera.

Stichorkis togensis (J.J.Sm.) Naive \& Ormerod, comb. nov.

Basionym: Liparis togensis J.J.Sm., Bull. Jard. Bot. Buitenzorg, sér. 3, 9: 457. 1928.

TYPE: INDONESIA. Maluku Prov., Buru, Gunung

Toga, 1900 m, February 1912, Stresemann 572 (holotype: L).

Distribution: Indonesia (Maluku Prov., Buru Island).

Stichorkis trullifera (Ames \& C.Schweinf.) Naive \& Ormerod, comb. nov.
Basionym: Liparis trullifera Ames \& C.Schweinf., Contr. Arn. Arb. 8: 26. 1934.

Synonym: Disticholiparis trullifera (Ames \& C.Schweinf.) Marg. \& Szlach., Orchidee (Hamburg) 55, 2: 179. 2004.

TYPE: INDONESIA. Sumatra, Kabajakan to Tretet trail, 1065-1525 m, 13 January 1932, Bangham 867 (holotype: AMES!; isotype: AMES!).

Distribution: Indonesia (Sumatra).

New combinations for East Malesian taxa

Stichorkis anceps (Schltr.) Ormerod \& Naive, comb. nov.

Basionym: Liparis anceps Schltr., Bot. Jahrb. Syst. 58: 65. 1922.

TYPE: PAPUA NEW GUINEA. Sepik District, Hunstein Peak, 1300 m, March 1913, Ledermann 11310 (holotype: B, destroyed).

Distribution: Papua New Guinea.

Stichorkis anemophila (Schltr.) Ormerod \& Naive, comb. nov.

Basionym: Liparis anemophila Schltr., Repert. Sp. Nov. Regni Veg., Beih. 1: 214. 1911.

Synonym: Disticholiparis anemophila (Schltr.) Marg. \& Szlach., Orchidee (Hamburg) 55, 2: 176. 2004.

TYPE: PAPUA NEW GUINEA. Ibo Range, 110 m, December 1917, Schlechter 17100 (syntype: $\mathrm{B}$, destroyed; isosyntypes: AMES!, BO, E, G 00354754, GH 00100778, K 000943205, L, MO, NSW!); Bismarck Range, $1400 \mathrm{~m}$, November 1909, Schlechter 18592 (syntype: B, destroyed; isosyntypes: G, L, S).

Distribution: Papua New Guinea.

Stichorkis apiculata (Schltr.) Ormerod \& Naive, comb. nov.

Basionym: Liparis apiculata Schltr., Repert. Sp. Nov. Regni Veg., Beih. 1: 216. 1911.

Synonym: Disticholiparis apiculata (Schltr.) Marg. \& Szlach., Orchidee (Hamburg) 55, 2: 176. 2004.

TYPE: PAPUA NEW GUINEA. Kani Range, 1000 m, September 1909, Schlechter 16552 (holotype: $\mathrm{B}$, destroyed; isosyntypes: $\mathrm{BM}, \mathrm{BO}, \mathrm{K}, \mathrm{L}, \mathrm{NSW}$ !, S).

Distribution: Papua New Guinea. 
Stichorkis arrigens (J.J.Sm.) Ormerod \& Naive, comb. nov.

Basionym: Liparis arrigens J.J.Sm., Nova Guinea 18: 25. 1935.

TYPE: INDONESIA. Papua Prov., Nassau Mts., Exploration Bivouac, $700 \mathrm{~m}$, October 1926, Docters van Leeuwen 10524 (holotype: BO; isotypes: $\mathrm{BO}, \mathrm{L}$ ).

Distribution: Indonesia ( Papua Prov.).

Stichorkis brunnescens (Schltr.) Ormerod \& Naive, comb. nov

Basionym: Liparis brunnescens Schltr., Repert. Sp.

Nov. Regni Veg., Beih. 1: 213. 1911.

Synonym: Disticholiparis brunnescens (Schltr.) Marg. \& Szlach., Orchidee (Hamburg) 55, 2: 176. 2004.

TYPE: PAPUA NEW GUINEA. Finisterre Range, 1300 m, January 1909, Schlechter 19102 (holotype: $\mathrm{B}$, destroyed).

Distribution: Papua New Guinea.

Stichorkis cyclostele (Schltr.) Ormerod \& Naive, comb. nov

Basionym: Liparis cyclostele Schltr., Repert. Sp. Nov.

Regni Veg., Beih. 1: 212. 1911.

Synonym: Disticholiparis cyclostele (Schltr.) Marg. \&

Szlach., Orchidee (Hamburg) 55, 2: 178. 2004.

TYPE: PAPUA NEW GUINEA. Waria River, near

Jaduna, 300 m, April 1909, Schlechter 19301

(syntype: B, destroyed; isosyntypes: AMES!, BO,

G, L, NSW!, S); Waria River, near Pema, May

1909, Schlechter s.n. (syntype: B, destroyed)

Distribution: Papua New Guinea.

Stichorkis finetiana (Schltr.) Ormerod \& Naive, comb. nov

Basionym: Liparis finetiana Schltr., Repert. Sp. Nov. Regni Veg. 10: 29. 1911.

Synonym: Liparis disticha auct. non (Thouars) Lindl.:

Schltr., Bot. Jahrb. Syst. 39: 60. 1906.

TYPE: NEW CALEDONIA. Near Paita, $200 \mathrm{~m}$,

October 1902, Schlechter 14858 (syntype: B, destroyed; isosyntypes: AMES! 00600518, K! 000943536, P, PR, WRSL); Mt. Iguambi, near Oubatche, $800 \mathrm{~m}$, December 1902, Schlechter 15489 (syntype: B, destroyed; isosyntype: P).

Distribution: New Caledonia; Fiji.
Stichorkis gautierensis (J.J.Sm.) Ormerod \& Naive, comb. nov.

Basionym: Liparis gautierensis J.J.Sm., Repert. Sp. Nov. Regni Veg. 11: 136. 1912.

TYPE: INDONESIA. Papua Prov., Gautier River, 700 m, November 1911, Gjellerup 875 (holotype: BO; isotype: L).

Distribution: Indonesia (Papua Prov.).

Stichorkis geelvinkensis (J.J.Sm.) Ormerod \& Naive, comb. nov.

Basionym: Liparis geelvinkensis J.J.Sm., Repert. Sp. Nov. Regni Veg. 12: 395. 1913.

Synonym: Disticholiparis geelvinkensis (Schltr.) Marg. \& Szlach., Orchidee (Hamburg) 55, 2: 178. 2004.

TYPE: INDONESIA. Papua Prov., Geelvink Bay, Giriwo River, July 1912, Janowsky 100 (holotype: $\mathrm{BO}$; isotype: $\mathrm{L}$ ).

Distribution: Indonesia (Papua Prov.).

Stichorkis gjellerupii (J.J.Sm.) Ormerod \& Naive, comb. nov.

Basionym: Liparis gjellerupii J.J.Sm., Repert. Sp. Nov. Regni Veg. 11: 557. 1912.

TYPE: INDONESIA. Papua Prov., Gautier Range, 400 m, November 1911, Gjellerup 881 (holotype: BO). Distribution: Indonesia (Papua Prov.).

Stichorkis glumacea (Schltr.) Ormerod \& Naive, comb. nov.

Basionym: Liparis glumacea Schltr., Repert. Sp. Nov. Regni Veg., Beih. 1: 219. 1911.

Synonym: Disticholiparis glumacea (Schltr.) Marg. \& Szlach., Orchidee (Hamburg) 55, 2: 178. 2004.

TYPE: PAPUA NEW GUINEA. Maboro Range, Govidjoa Creek, 1200 m, June 1909, Schlechter 19805 (holotype: B, destroyed).

Distribution: Papua New Guinea.

Stichorkis govidjoae (Schltr.) Ormerod \& Naive, comb. nov.

Basionym: Liparis govidjoae Schltr., Repert. Sp. Nov.

Regni Veg., Beih. 1: 218. 1911.

Synonym: Disticholiparis govidjoae (Schltr.) Marg. \&

Szlach., Orchidee (Hamburg) 55, 2: 178. 2004.

TYPE: PAPUA NEW GUINEA. Govidjoa Creek, 
1200 m, June 1909, Schlechter 19083 (holotype: B, destroyed).

Distribution: Papua New Guinea.

Stichorkis graciliscapa (Schltr.) Ormerod \& Naive, comb. nov.

Basionym: Liparis graciliscapa Schltr., Repert. Sp.

Nov. Regni Veg., Beih. 1: 211. 1911.

Synonym: Disticholiparis graciliscapa (Schltr.) Marg.

\& Szlach., Orchidee (Hamburg) 55, 2: 178. 2004.

TYPE: PAPUA NEW GUINEA. Djamu Gorge, $450 \mathrm{~m}$,

November 1907, Schlechter 16807 (holotype: B, destroyed; isotypes: L, NSW!, S).

Distribution: Papua New Guinea.

Stichorkis inamoena (Schltr.) Ormerod \& Naive, comb. nov.

Basionym: Liparis inamoena Schltr., Repert. Sp. Nov.

Regni Veg., Beih. 1: 216. 1911.

Synonym: Disticholiparis inamoena (Schltr.) Marg. \&

Szlach., Orchidee (Hamburg) 55, 2: 178. 2004.

TYPE: PAPUA NEW GUINEA. Bismarck Range,

1600 m, November 1908, Schlechter 18784

(holotype: B, destroyed).

Distribution: Papua New Guinea.

Stichorkis janowskyi (J.J.Sm.) Ormerod \& Naive, comb. nov.

Basionym: Liparis janowskyi J.J.Sm., Repert. Sp. Nov.

Regni Veg. 12: 395. 1913 (as 'janowskii', corrected

by Smith in Nova Guinea 12(3): 230. 1915).

TYPE: INDONESIA. Papua Prov., Giriwo River, July

1912, Janowsky 210 (holotype: BO).

Distribution: Indonesia (Papua Prov.).

Stichorkis lamproglossa (Schltr.) Ormerod \& Naive, comb. nov.

Basionym: Liparis lamproglossa Schltr., Repert. Sp.

Nov. Regni Veg., Beih. 1: 220. 1911.

Synonym: Disticholiparis lamproglossa (Schltr.)

Marg. \& Szlach., Orchidee (Hamburg) 55, 2: 178. 2004.

TYPE: PAPUA NEW GUINEA. Finisterre Range,

1100 m, November 1908, Schlechter 18625

(holotype: B, destroyed; isotypes: AMES!, BO, G,

L, NSW!, S).

Distribution: Papua New Guinea.
Stichorkis loliacea (Ridl.) Ormerod \& Naive, comb. nov.

Basionym: Liparis loliacea Ridl., Trans. Linn. Soc. s.2, Bot. 9: 164. 1916.

TYPE: INDONESIA. Papua Prov., W bank of Tsingarong River, Camp 6A, 945 m, January 1913, Kloss s.n. (holotype: BM).

Distribution: Indonesia (Papua Prov.).

Stichorkis miniata (Schltr.) Ormerod \& Naive, comb. nov.

Basionym: Liparis miniata Schltr., Repert. Sp. Nov. Regni Veg., Beih. 1: 218. 1911.

Synonym: Disticholiparis miniata (Schltr.) Marg. \& Szlach., Orchidee (Hamburg) 55, 2: 178. 2004.

TYPE: PAPUA NEW GUINEA. Govidjoa Creek, 1000 m, June 1909, Schlechter 19838 (holotype: B, destroyed).

Distribution: Papua New Guinea.

Stichorkis murkelensis (J.J.Sm.) Naive \& Ormerod, comb. nov.

Basionym: Liparis murkelensis J.J.Sm., Bull. Jard. Bot. Buitenzorg, sér. 3, 10: 127. 1928.

TYPE: INDONESIA. Maluku Prov., Seram, Gunung Moerkele, 2000-2500 m, 1 July 1918, Rutten 1483 (syntype: L); Gunung Moerkele, 2000-2500m, 1 July 1918, Rutten 1491 (syntype: L).

Distribution: Indonesia (Maluku Prov., Seram Island).

Stichorkis nebuligena (Schltr.) Ormerod \& Naive, comb. nov.

Basionym: Liparis nebuligena Schltr., Repert. Sp. Nov. Regni Veg., Beih. 1: 212. 1911.

Synonym: Disticholiparis nebuligena (Schltr.) Marg. \& Szlach., Orchidee (Hamburg) 55, 2: 178. 2004.

TYPE: PAPUA NEW GUINEA. Torricelli Range, near Akur, 700 m, September 1909, Schlechter 20112 (syntype: B!); Kani Range, 1000 m, December 1907, Schlechter 16961 (syntype: B, destroyed; isosyntypes: AMES!, BO, E, G, GH, K, L, MO, NSW, S); Maboro Range, 1300 m, May 1909, Schlechter 19514 (syntype: B, destroyed).

Distribution: Papua New Guinea.

Stichorkis ochrantha (Schltr.) Ormerod \& Naive, comb. nov. 
Basionym: Liparis ochrantha Schltr., Repert. Sp. Nov. Regni Veg., Beih. 1: 214. 1911.

Synonym: Disticholiparis ochrantha (Schltr.) Marg. \& Szlach., Orchidee (Hamburg) 55, 2: 178. 2004.

TYPE: PAPUA NEW GUINEA. Minjem Valley, Kelel, 400 m, July 1907, Schlechter 16308 (syntype: B!; isosyntypes: AMES!, BM, G , K, L, NSW, S); near Ambo, 500 m, September 1908, Schlechter 18260 (syntype: B, destroyed; isosyntypes: AMES!, BM, BO, E, GM, K, L, NSW!, S).

Distribution: Papua New Guinea.

Stichorkis pandaneti (J.J.Sm.) Ormerod \& Naive, comb. nov.

Basionym: Liparis pandaneti J.J.Sm., Nova Guin. 12: 16. 1913.

TYPE: INDONESIA. Papua Prov., Noord River, September 1907, Versteeg 1743 (syntype: BO); same area, September 1909, von Roemer 156 (syntype: BO); same area, October 1911, von Roemer 440 (syntype: BO).

Distribution: Indonesia (Papua Prov.).

Stichorkis pseudodisticha (Schltr.) Ormerod \& Naive, comb. nov.

Basionym: Liparis pseudodisticha Schltr., in Schum.

\& Laut., Fl. Deutsch. Schutzgeb. Südsee, Nachtr. 2: 106.1905.

Synonym: Disticholiparis pseudodisticha (Schltr.) Marg. \& Szlach., Orchidee (Hamburg) 55, 2: 179. 2004.

TYPE: PAPUA NEW GUINEA. Bismarck Range, 1200 m, January 1902, Schlechter 14064 (holotype: $\mathrm{B}$, destroyed; istotypes: BM, BRI, K, P).

Distribution: Indonesia (Papua Prov.); Papua New Guinea.
Stichorkis trachyglossa (Schltr.) Ormerod \& Naive, comb. nov.

Basionym: Liparis trachyglossa Schltr., Repert. Sp. Nov. Regni Veg., Beih. 1: 217. 1911.

Synonym: Disticholiparis trachyglossa (Schltr.) Marg. \& Szlach., Orchidee (Hamburg) 55, 2: 179. 2004. TYPE: PAPUA NEW GUINEA. Torricelli Range, 800 m, September 1909, Schlechter 20338 (holotype: $\mathrm{B}$, destroyed; isotypes: $\mathrm{BM}, \mathrm{BO}, \mathrm{K}, \mathrm{L}, \mathrm{NSW}$ !, S). Distribution: Papua New Guinea.

Stichorkis triticea (Ridl.) Ormerod \& Naive, comb. nov.

Basionym: Liparis triticea Ridl., Trans. Linn. Soc. s.2, Bot. 9: 164. 1916.

TYPE: INDONESIA. Papua Prov., Camp 1, 215 m, November/December 1912, Kloss s.n. (syntype: BM); W bank of Tsingarong River, Camp 6A, 945 m, 15 January 1913, Kloss s.n. (syntype: BM).

Distribution: Indonesia (Papua Prov.).

Stichorkis togensis (J.J.Sm.) Naive \& Ormerod, comb. nov.

Basionym: Liparis togensis J.J.Sm., Bull. Jard. Bot. Buitenzorg, sér. 3, 9: 457. 1928.

TYPE: INDONESIA. Maluku Prov., Buru, Gunung

Toga, 1900 m, February 1912, Stresemann 572 (holotype: L).

Distribution: Indonesia (Maluku Prov., Buru Island).

Acknowledgements. We would like to thank Dr. Pieter Pelser, Dr. Julie Barcelona and Mr. Ravan Schneider for allowing us to use their photos. The second author wishes to thank the herbarium and library staff at the Harvard University Herbaria for their help and hospitality during his visits.

\section{LiTERATURE CITED}

Ames, O. (1905). Orchidaceae: Illustration and Studies of the Family Orchidaceae. Cambridge: Houghton, Mifflin and Co. Riverside Press. 156 pp.

Cootes, J. (2011). Philippine native orchid species. Quezon City: Katha Publishing Co.

Naive, M. A. K., Cootes, J. \& Ormerod, P. (2019). Stichorkis davidlohmanii (Orchidaceae; Malaxideae), a new species from the southern Philippines. Taiwania, 64(1), 65-68. doi: 10.6165/tai.2019.64.65

Pelser, P. B., Barcelona, J. F. \& Nickrent, D. L. (Eds.). (2011 onwards). Co's Digital Flora of the Philippines. Retrieved from www. philippineplants.org [Accessed 2 September 2018].

Turland, N. J., Wiersema, J. H., Barrie, F. R., Greuter, W., Hawksworth, D. L., Herendeen, P. S., Knapp, S., Kusber, W.-H., Li, D.-Z., Marhold, K., May, T. W., McNeill, J., Monro, A. M., Prado, J., Price, M. J. \& Smith, G. F. (Eds.). (2018). 
International Code of Nomenclature for algae, fungi, and plants (Shenzhen Code) adopted by the Nineteenth International Botanical Congress Shenzhen, China, July 2017. Regnum Vegetabile 159. Glashütten: Koeltz Botanical Books. DOI: https://doi.org/10.12705/Code.2018 
